\title{
Comparison of techniques for the estimation of flow parameters of fan inflow turbulence from noisy hot-wire data
}

\author{
Luciano Caldas, Carolin Kissner, Maximilian Behn, Ulf Tapken, Robert Meyer \\ German Aerospace Center (DLR), Institute of Propulsion Technology, Engine Acoustics Department, Berlin, Germany
}

Precise estimation of flow turbulence parameters such as integral length scale (ILS) and turbulence intensity ( Tu) is a key requirement for many aerodynamic and aeroacoustic analyses. The assessment of these parameters are often done on hot-wire measurement data. For several reasons this measurement technique is sensitive to external contamination in its recorded signal. If not addressed correctly, this problem may lead to large errors in the estimation of turbulence parameters. This paper proposes a signal processing chain performed partially in the time and in the frequency domain to remove or reduce disturbances in the recorded instationary velocity signal, which are not expected to be turbulence related. Two turbulence spectrum models - von-Kármán and Bullen - are fitted to the estimated spectrum from measured data. Parameters estimated via several different techniques are compared. Hot-wire data measured in a high-speed (Mach $=0.48$ ) fan rig are used. The behavior of both ILS and Tu levels are plotted for two fan speeds and several radial positions. Most of techniques converge to a comparable value of ILS and Tu. Fitted spectra often match the estimated spectrum from measured data. The estimation techniques also enabled the investigation of ILS and Tu ratios, which indicated that the turbulence has an anisotropic characteristic at all investigated probe positions and fan speeds.

\section{Nomenclature}
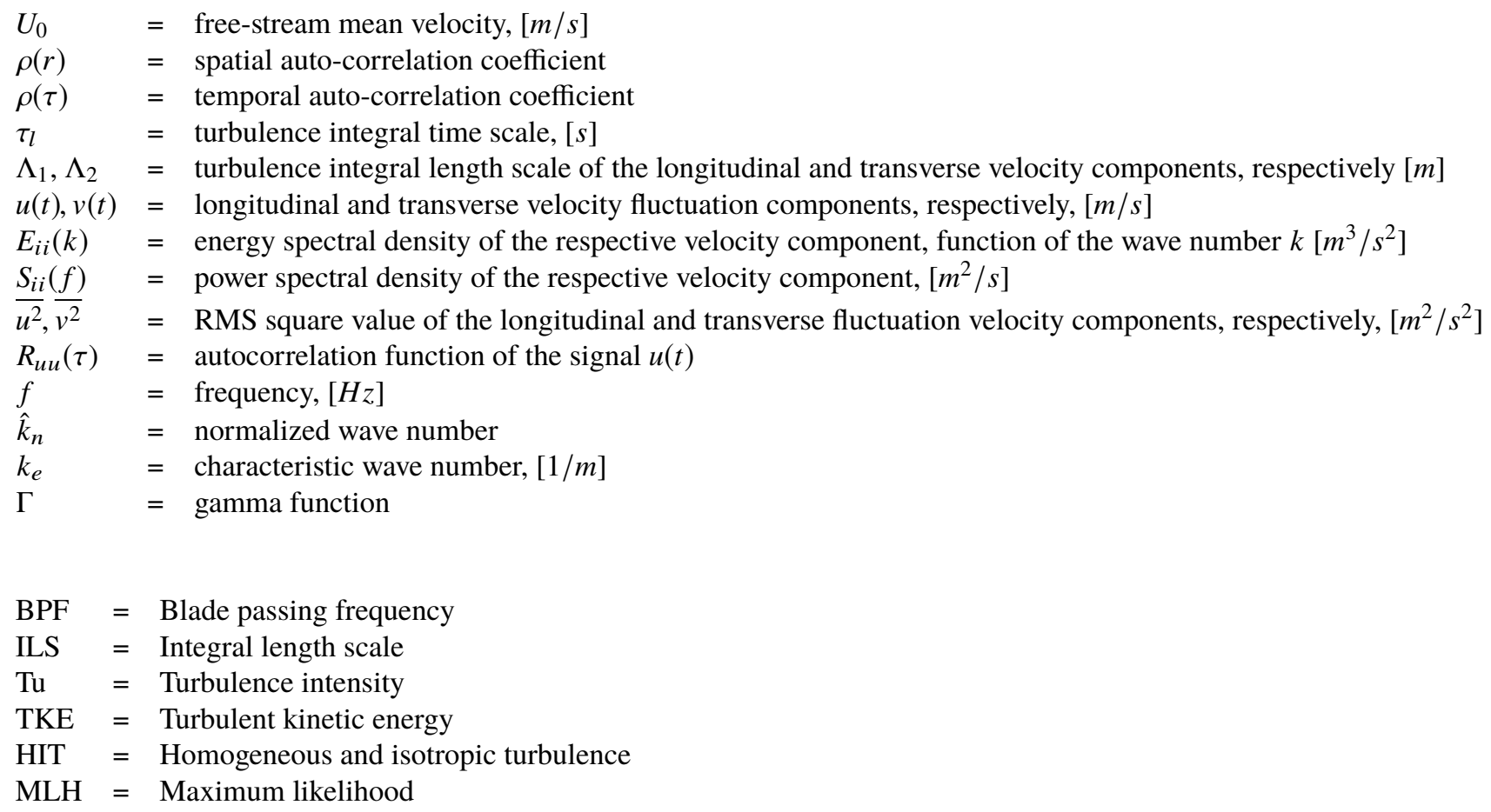


\section{Introduction}

$\mathrm{T}$ URBULENCE parameters such as turbulence intensity and turbulence length scale describe the statistically averaged behavior of turbulence structures in the flow. Turbulence intensity and length scale are known as having a strong influence on airfoil aerodynamics, such as on the boundary layer thickness and transition [1], on turbine aerodynamics [2], on heat transfer [3, 4], and also on the acoustic emissions of an airfoil (or similarly of a rotor/stator set) [5- -8].

The main focus of the present work is to improve techniques for the estimation of turbulence parameters. Commonly used methods for fan broadband prediction, which include analytic models [8-11], synthetic turbulence methods [12, 13], and scale resolving techniques [14, 15], rely on correctly estimated turbulence parameters to be used as input as validation data. Required input and validation parameters can include variables such as the boundary layer thickness, turbulence kinetic energy, and the turbulence integral length scale at the fan inflow or in the fan interstage domain. Therefore, the accurate estimation of these parameters from experimental data is crucial to ensure more accurately predicted noise levels. In this work, turbulence kinetic energy and turbulence ILS are assessed from hot-wire anemometry data.

Hot-wire measurement is a complicated, sensitive and time demanding technique. The thin wires (typically about 2.8 $\mathrm{mm}$ long and $9-12.5 \mu \mathrm{m}$ in diameter [16]) are held by a probe and intrusively inserted into the flow. The measurement is performed point-wise and therefore the probe needs to be traversed in order to obtain a radial measurement profile, or to be traversed in both radial and circumferential direction, if the total cross-section evaluation is needed.

One of the several complications in such measurement is the contamination of the electrical signals from the hot-wires due to different sources. In this context we can highlight two of them: electronic/electrical interference and - vibration in the probe excited by the rotating fan/shaft/motor. The first can be usually easier to deal with, as it normally excites pure tones and their harmonics in the signal, typically originating from the grid, motor controller, etc. The second effect is potentially more complicated, because the test rig excites vibration in the whole traverse/probe/wire mechanisms, and the frequency of this excitement could be orders higher or lower than the source of vibration. Besides that, there are also resonance frequencies with respect to the wire and/or the probe system, which could also be excited by the fan rig vibration.

This paper proposes a signal processing chain procedure to filter out distortions (non turbulence related) from the measured hot-wire velocity signals, followed by different proposed estimation techniques of turbulence parameters (such as turbulence intensity and turbulence integral length scale), most of which are based on turbulence spectrum models. The paper is structured as follows:

- Section[III briefly discusses turbulence fundamentals, the length-scale problem, the integral length scale and two spectrum models.

- Section IV]describes the signal processing chain proposed in our work to deal with contaminated data.

- Section V shows a performance test of all estimators with synthetic data superposed by distortions.

- SectionVI describes the experimental setup.

- Experimental data are shown and discussed in Section VII

- The paper ends with conclusions and remarks in Section VIII

\section{A brief overview of relevant turbulence characteristics}

Turbulence is a vast, interdisciplinary and complex field of study. As argued by Tennekes [17] “...turbulence theory suffers from the absence of sufficiently powerful mathematical methods. This lack of tools makes all theoretical approaches to problems in turbulence trial-and-error affairs". Along with that, one can state that due to the strong stochastic (non deterministic) behavior of turbulence, statistical tools are used in order to extract any useful information from instationary flow velocity data. In the context of this work, instationary velocity data refers to a time-series signal with a sampling frequency of several kilo-hertz. According to Hinze [18] (in agreement also with Tennekes [17]), “...turbulence is a multiple length scale problem. Scales are bounded from above (large scales) by the dimensions of the flow field and bounded from below (small scales) by the diffusive action of molecular viscosity. That is the reason spectral analyses of turbulent motions are so useful". In other words, a wide range of turbulence structure sizes are present in a given flow. When the turbulence is found in equilibrium, large structures break down and fed its energy cascaded into smaller structures. This process continues until scales are small enough to dissipate their energy via viscosity (and become thermal energy). Richardson [19] even wrote that in a more poetic way 
"Big whorls have little whorls,

Which feed on their velocity;

And little whorls have lesser whorls,

And so on to viscosity"

Apart from poems, it is desired to establish some metric to quantify the statistically averaged (in some sense) size of the turbulence structures. A commonly adopted metric is the turbulence integral length scale (ILS). This metric aims to estimate the size of the most energetic eddies in a turbulent flow, as briefly discussed in the next section.

\section{A. Turbulence integral length scale - ILS}

The turbulence integral length scale is assessed by the integral of the spatial velocity coherence

$$
\Lambda=\int_{-\infty}^{\infty} \rho(r) d r
$$

where $\rho(r)$ is the cross-correlation coefficient between two points separated by a distance $r$ in the flow-field. To experimentally determine the spatial correlation defined in Eq. 4. simultaneous measurements are required from two probes and the distance between them needs to be varied. Because of physical constraints, such measurements are not preferred, or even impossible to realize. Instead, the spatial correlation is approximated by temporal auto-correlation employing Taylor's hypothesis [18] often also denoted as the "frozen-turbulence" assumption. The integral time scale (ITS, $\tau_{l}$ ) can be obtained by the temporal auto-correlation coefficient according to

$$
\tau_{1}=\int_{-\infty}^{\infty} r_{11}(\tau) d \tau,
$$

where the auto-correlation coefficient $r_{u u}(\tau)$ is obtained from the velocity fluctuation signal $u(t)$

$$
r_{11}(\tau)=\frac{\overline{u(t) u(t-\tau)}}{\overline{u^{2}}}=\frac{R_{11}(\tau)}{R_{11}(0)}
$$

The overbar stands for the time-mean value. Finally, the ILS can be estimated under Taylor's hypotheses by

$$
\Lambda_{1}=\tau_{1} U_{0}
$$

where $U_{0}$ stands for the mean flow speed.

The auto-correlation function can alternatively be obtained by the inverse Fourier transform of the power spectral density $S_{u u}(f)$ of the velocity fluctuation $u(t)$

$$
r_{11}(\tau)=\frac{1}{\overline{u^{2}}} \int_{0}^{\infty} S_{11}(f) e^{j 2 \pi f t} d f
$$

on the other hand, we know by the Parseval Theorem [20] that

$$
R_{11}(0)=\int_{-\infty}^{\infty} S_{11}(f) d f
$$

and

$$
S_{11}(0)=\int_{-\infty}^{\infty} R_{11}(\tau) d \tau
$$

and therefore the integral time scale can be obtained by

$$
\tau_{1}=\frac{S_{11}(0)}{\overline{u^{2}}}
$$

where

$$
\overline{u^{2}}=\int_{-\infty}^{\infty} S_{11}(f) d f
$$

By assuming the Taylor hypotheses, we finally obtain

$$
\Lambda_{1}=\tau_{1} U_{0}=\frac{S_{11}(0) U_{0}}{\overline{u^{2}}}
$$




\section{B. Integral length scale under the assumption of homogeneous and isotropic turbulence (HIT)}

Hinze [18] defines the condition of isotropy as "by the invariance under rotation of the coordinate system and under reflection with respect to the coordinate planes of the statistically averaged properties of the turbulence." A more complete definition is given by Batchelor [21] "In isotropic turbulence the joint-probability distribution of the velocities at any arbitrarily chosen $n$ points in space is invariant under arbitrary rotations of the configuration as formed by the $n$ points and by the various direction vectors, and under reflection of the configuration with respect to any plane". A closer discussion of HIT is, however, outside the scope of this work. Instead, a hint of isotropy is assessed based on the ratio of turbulence intensity and integral length scale for the axial and longitudinal velocity components. As a matter of reducing complexity, isotropic turbulence spectrum models are used in this work.

For the sake of assessing the integral length scale (ILS), by assuming isotropic and homogeneous turbulence, the velocity spectrum can be modelled as showed by Roach [22] as

$$
\frac{4 \overline{u^{2}} \Lambda_{1}}{S_{11}(f) U_{0}}=1+\left(\frac{2 \pi f \Lambda_{1}}{U_{0}}\right)^{2}
$$

and therefore

$$
\Lambda_{1}=\left[\frac{S_{11}(f) U_{0}}{4 \overline{u^{2}}}\right]_{f \rightarrow 0}
$$

whereas for the transverse component it holds

$$
\Lambda_{2}=\left[\frac{S_{22}(f) U_{0}}{2 \overline{v^{2}}}\right]_{f \rightarrow 0}
$$

We note that the factor 2 between these two scales agrees with the theoretical relation between the longitudinal and transverse integral scales Hinze [18] Eqs 3-74 and 3-75 for isotropic turbulence, i.e., $\Lambda_{2}=\Lambda_{1} / 2$.

As it will be introduced in the next section regarding turbulence spectrum models, equations 11 and 12 can be verified by setting $f=0$ or equivalently $\hat{k}_{n}=0$ onto equations 13 and 14 for example. At this point an attuned reader might ask him- or herself why equations 9 and 11 are so similar, except the factor 4 dividing Eq. 11. Indeed the turbulence spectrum when $f=0$ (which physically has a meaning similar to a RMS value) is not the same as for the nearby low-frequency bins $f \rightarrow 0$ (which is basically the power density of the very low-frequency components). This fact is (from the author's understanding) not modeled by isotropic turbulence models, but it is observed on measured turbulence data.

\section{Isotropic and homogeneous turbulence spectrum models}

\section{The 2 parameters von-Kármán spectrum model}

A suitable turbulence model often used in the literature for turbulence modeling is the one proposed by von-Kármán. The two-dimensional energy spectrum for the longitudinal and transverse velocity component is defined [18, 23] as

$$
\begin{aligned}
& E_{11}=\frac{\overline{u^{2}} \Lambda_{1}}{\pi} \frac{1}{\left(1+\hat{k}_{n}^{2}\right)^{5 / 6}} \\
& E_{22}=\frac{\overline{u^{2}} \Lambda_{1}}{2 \pi} \frac{1+\frac{8}{3} \hat{k}_{n}^{2}}{\left(1+\hat{k}_{n}^{2}\right)^{11 / 6}}
\end{aligned}
$$

where the subscript 11 and 22 stand for the longitudinal and transverse velocity component, respectively. The other variables are described below. 


\section{The 3 parameters Bullen spectrum model}

The Bullen spectrum [24, 25] can be seen as a modified von-Kármán spectrum, where the decaying rate of it can be adjusted by the variable $n$. When $n=1 / 3$ it reduces to the von-Kármán spectrum.

$$
\begin{gathered}
E_{11}=\frac{\overline{u^{2}} \Lambda_{1}}{\pi} \frac{1}{\left(1+\hat{k}_{n}^{2}\right)^{n+1 / 2}} \\
E_{22}=\frac{\overline{u^{2}} \Lambda_{1}}{2 \pi} \frac{1+2(n+1) \hat{k}_{n}^{2}}{\left(1+\hat{k}_{n}^{2}\right)^{n+3 / 2}},
\end{gathered}
$$

where $\hat{k}_{n}$ is the normalized wave-number given by

$$
\hat{k}_{n}=\hat{k}\left(f, n, \Lambda_{1}\right)=\frac{2 \pi f}{v_{0} k_{e}\left(n, \Lambda_{1}\right)}
$$

and

$$
k_{e}\left(n, \Lambda_{1}\right)=\frac{\sqrt{\pi} \Gamma(n+1 / 2)}{\Lambda_{1} \Gamma(n)}
$$

$\Gamma(n)$ is the Gamma function of order $n$. For the von-Kármán spectrum $n$ should be set to $1 / 3$.

The power spectral density of the turbulence velocity can be finally obtained by

$$
S_{i i}(f)=\frac{4 \pi}{U_{0}} E_{i i},
$$

where the subscript 'ii' stands for the respective velocity component.

\section{Spectrum test case}

Fig. 11 shows a test case for both spectrum models, as well as for both longitudinal and transverse velocity component. Absolute and normalized plots are displayed. Bullen spectrum is plotted with two different falling rate $n=1$ and $n=3$. These two examples feature a steeper decay at high frequencies compared to the von-Kármán spectrum, where $n=1 / 3$.

\section{Parameter estimation and signal processing techniques}

A signal processing chain is proposed in this work. The following subsections IV.A and IV.B are used to pre-process the signal and to remove as much disturbances as possible. Subsequently, three slightly different techniques are applied to estimate turbulence parameters: Two spectrum fit techniques are discussed in subsections IV.C.and IV.D A maximum likelihood estimation technique is in short described in subsection IV.E

\section{A. Data resampling and cyclostationary analysis}

The velocity signals obtained by the hot-wire anemometer is first linearly re-sampled with reference to the rotor shaft trigger. This is followed by a cyclostationary analysis [26, 27], which splits the rotor coherent and incoherent signal constituents. The inflow turbulence is expected to be incoherent with the fan rotating frequency, whereas all vibration sources and blade passing frequency (BPF) related tones are coherent. The incoherent signal component (CyS2) is then used as input for the subsequent steps of the signal processing chain.

\section{B. Low-frequency disturbance removal}

The applied technique was first developed and described by Mark [28], where hot-wire data was recorded from a probe mounted on an airplane wing. They observed that non-Gaussian low-frequency disturbances was present in the data. This disturbance was associated with "wind-gusts" and not related to turbulence itself. In our case, after the analysis of a large set of hot-wire data measured in the inlets of turbo-machines, it seems that the mechanisms of low-frequency disturbances encountered by Mark are similar to the one observed for this case.

In short, Mark [28] assumed that two random processes are mixed: so called "wind-gusts", which are characterized by low-frequency or slow oscillations, and turbulence, which is characterized by high-frequency or fast oscillations. Mathematically it can be formulated as follows: 


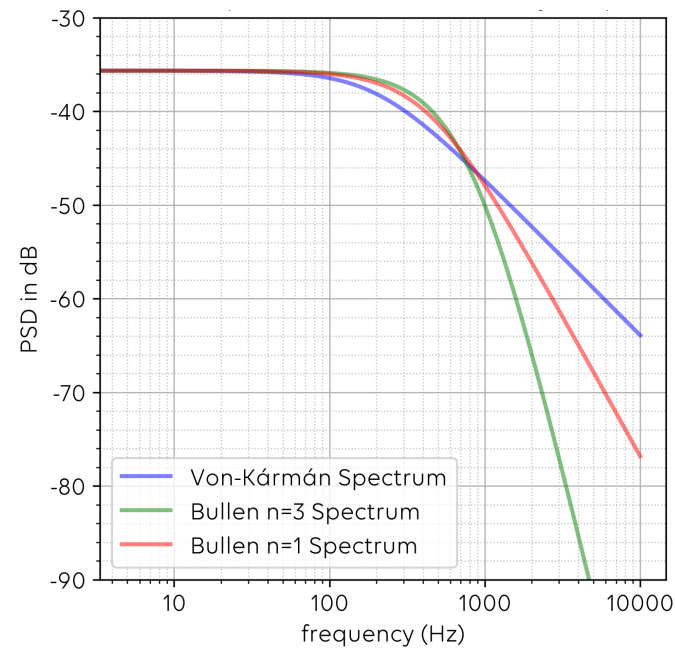

(a) Longitudinal velocity component $S_{11}(f)$.

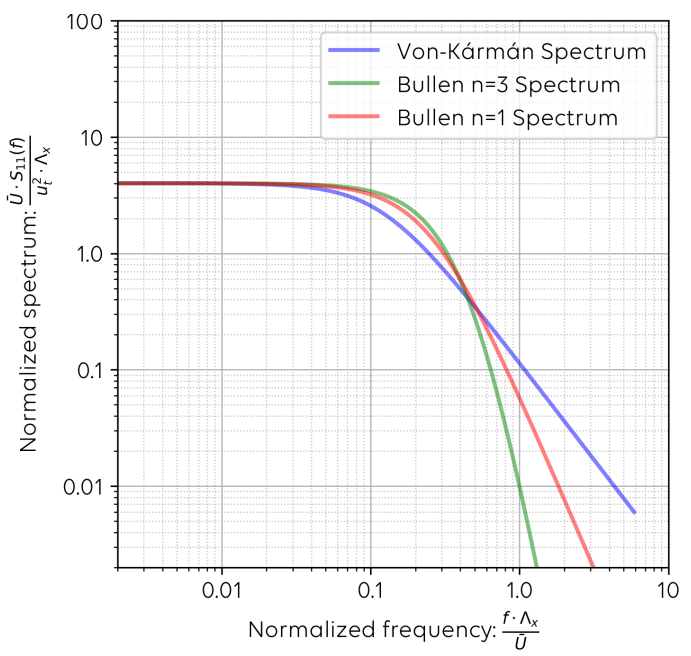

(c) Longitudinal velocity component $S_{11}(f)$.

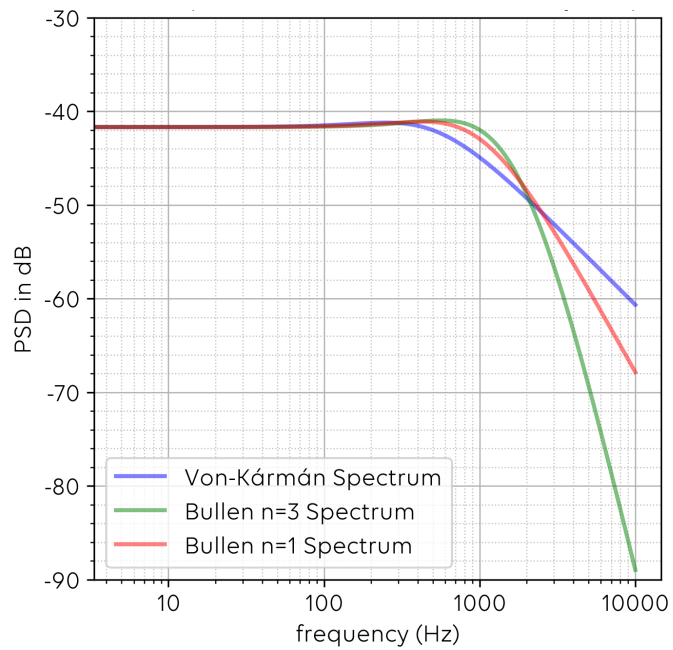

(b) Transverse velocity component $S_{22}(f)$.

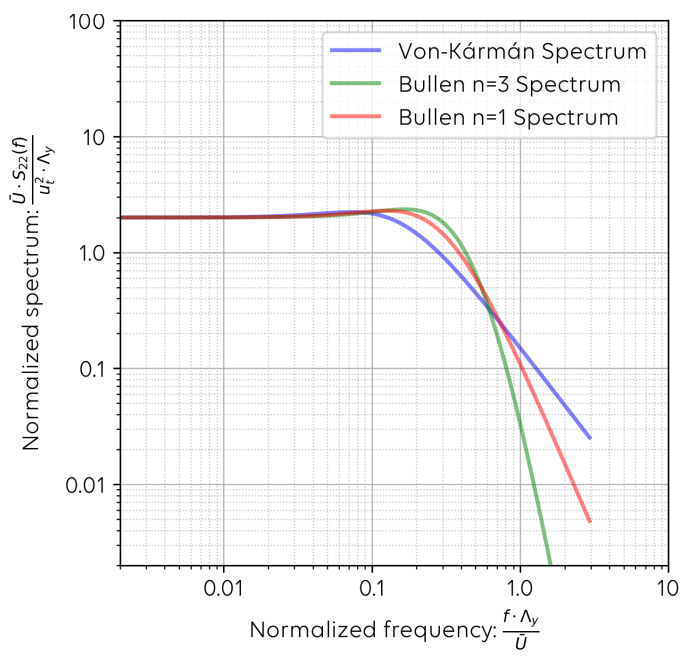

(d) Transverse velocity component $S_{22}(f)$.

Fig. 1 Synthetic turbulence spectrum for both axial and transverse velocity component. Parameters used: $U_{0}$ $=34 \mathrm{~m} / \mathrm{s}, T u=1 \%, I L S=20 \mathrm{~mm}$. Different turbulence models are depicted.

$$
\begin{aligned}
w(t) & =w_{s}(t)+w_{f}(t) \\
& =w_{s}(t)+\sigma_{f}(t) z(t),
\end{aligned}
$$

where $\sigma_{f}(t) z(t) \geq 0, E\{z(t)\}=\overline{z(t)}=0$ and $E\left\{z^{2}(t)\right\}=\overline{z^{2}(t)}=1$. In Eq. $16 w(t)$ denotes the measured signal. $w_{s}(t)$ is the "slow" (low-frequency) components and $w_{f}(t)$ the "fast" ones. Mark [28] further assumes that $z(t)$ is a Gaussian stationary process, and that $\sigma_{f}(t)$ is a stationary random process, but since $\sigma_{f}(t) z(t) \geq 0$, it is not assumed to be Gaussian. More importantly, for the proposed analysis it is assumed that $w_{s}(t)$ and $w_{f}(t)$ are statistically independent. Under this assumption the auto-correlation and the power spectral density can be obtained as

$$
R_{w}(\tau)=R_{w_{s}}(\tau)+R_{w_{f}}(\tau)
$$

and

$$
S_{w}(f)=S_{w_{s}}(f)+S_{w_{f}}(f) .
$$




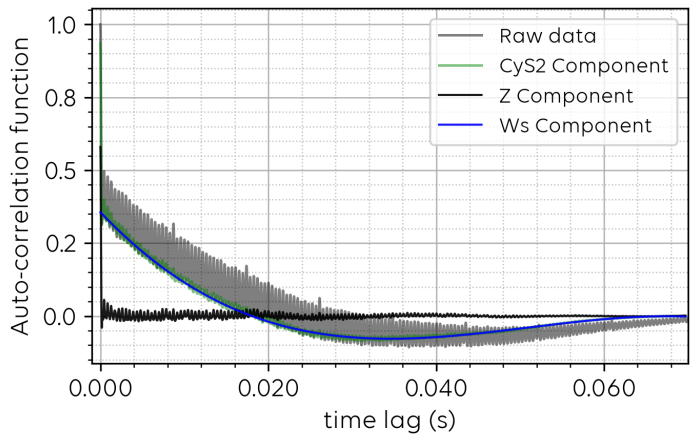

(a) Auto-correlation function and fitted polynomial interpolation.

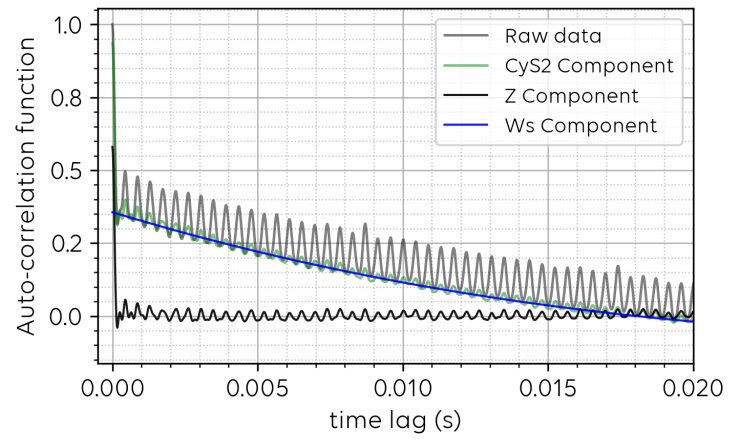

(b) Zoomed auto-correlation function.

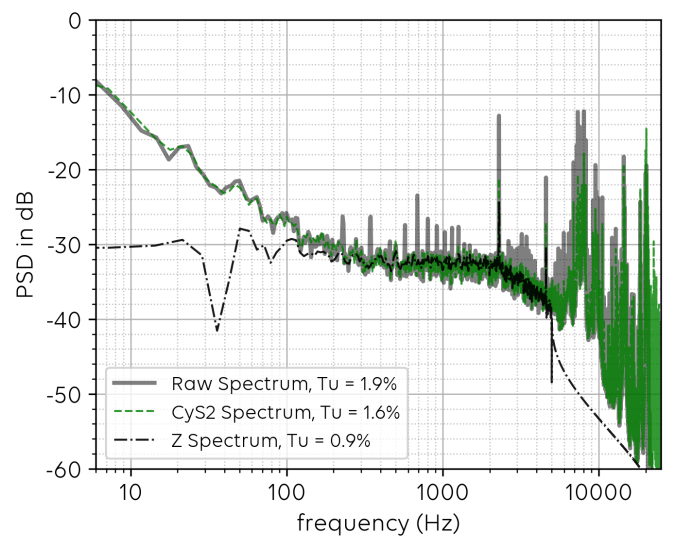

(c) Power spectral density before and after the low-frequency removal.

Fig. 2 Low-frequency disturbance removal technique test.

In order to implement the techniques proposed by Mark [28], the following steps were taken for the signal processing chain:

- Data re-sampling using a shaft-trigger signal

- Cyclostationary analysis. The CyS2 component (rotor-incoherent part of the signal) is selected as data input

- Power spectral density estimated via Welch [20, 29] method. The spectrum is limited to up to $\mathrm{f}=5 \mathrm{kHz}$. This can be attributed to the high noise incidence in the signal in this frequency range, as will be shown later.

- The auto-correlation function is then estimated as the inverse Fourier transform of the power spectral density. This auto-correlation function is then used as input for the above mentioned low-frequency removal technique.

By following the guidelines proposed by Mark [28], the "slow-component" of the auto-correlation function $R_{w_{s}}(\tau)$ can be interpolated as polynomial of order 2 or 3 . Figs. $2 \mathrm{a}$ and $2 \mathrm{~b}$ show the result of the technique applied to experimental data. Note that it matches very well with the result obtained by Mark [28] in Fig. 5 of that work. The green curve in Figs. $2 \mathrm{a}$ and $2 \mathrm{~b}$ "CyS2 Component" is used as input data and represents the auto-correlation function $R_{w}(\tau) . R_{w_{s}}(\tau)$ (blue curve) is the result of an order 3 polynomial interpolation to $R_{w}(\tau)$. The black curve stands for $R_{w_{f}}(\tau)$. The difference in decaying time comparing the blue "slow-component" and black curve "fast-components" is clearly seen in Fig. $2 \mathrm{~b}$ Finally, Fig. $2 \mathrm{c}$ shows the power spectral density of each component: raw signal, CyS2 component $\left(S_{w}(f)\right)$ and the turbulence resultant component $S_{w_{f}}(f)$.

\section{L-1 norm spectrum fit}

The idea behind this technique is to fit a turbulence spectrum to an estimated spectrum from measured data. A suitable turbulence model needs to be chosen. In this work, as showed in Section III.C the von-Kármán and the Bullen turbulence spectrum were selected, which are indeed constrained by the HIT assumption. By investigating differences 
in the turbulence characteristics obtained by fitting the longitudinal and transverse velocity components, conclusions regarding the validity of the assumption of HIT can be drawn.

The optimization problem is formulated as follows:

$$
\underset{\overline{u^{2}}, \Lambda_{1}, n \in \mathbb{R}}{\operatorname{argmin}}\left\|S(f)-S_{11}\left(f, \overline{u^{2}}, \Lambda_{1}, n, U_{0}\right)\right\|_{1},
$$

where $S(f)$ is the estimated spectrum from measured data (in our case, the output spectrum "Z Spectrum" obtained from the previous technique). Eq. 19 is formulated for the longitudinal velocity component, but it is analogously formulated for the transverse component. If the von-Kármán model is selected, then $n=1 / 3$. For the Bullen model, the optimal $n$ is automatically searched.

Another crucial parameter of this minimization problem is the norm selected as an objective criteria. Often the L2-norm is used as the typical least-squared-fit (LSF) problem, in order to minimize the residual power. For this approach however, the L1-norm is used in order to increase the weighting of the broadband components and decrease the importance attributed to the tonal components, which are known as non turbulence source [18, 30]. The impact of the chosen norm will be further discussed in Section $\mathrm{V}$. The choice of the L1-norm brings robustness against tonal components, often present in the measured data. This latter component, if not removed from the spectrum, leads to strong deviations in the turbulence parameter estimation, especially for the turbulence intensity, or equivalently the turbulent kinetic energy (TKE).

\section{L-1 norm spectrum fit with constraint}

This fit works similar to the previous one, except that now a constraint is added to the minimization problem, which is described as

$$
\begin{aligned}
& \underset{u^{2}, \Lambda_{1}, n \in \mathbb{R}}{\operatorname{argmin}}\left\|S(f)-S_{11}\left(f, \overline{u^{2}}, \Lambda_{1}, n, U_{0}\right)\right\|_{1} \\
& \text { subjected to } \Lambda_{1}=\left[\frac{S(f) U_{0}}{4 \overline{u^{2}}}\right]_{f \rightarrow 0}
\end{aligned}
$$

i.e., the variables $\overline{u^{2}}$ and $\Lambda_{1}$ now fulfill a physical constraint established by Eq. 11 This restriction reduces the degree of freedom of this fit technique by one degree. This restriction helps the convergence of the technique, especially when the data-set is highly contaminated.

\section{E. Maximum Likelihood estimator - von-Kármán turbulence model}

The last parameter estimation technique tested in the present work was described by Mark [25]. Maximum likelihood estimator is a robust statistical tool [20,29] to estimate parameters of a given model from data embedded with noise. For practicality of the reader, the formulation proposed by [25] is succinctly shown as follows. The formulation is provided for the von-Kármán turbulence model. Mark also showed the formulation for the Bullen model [24] but this was outside the scope of this work.

The two main equations to be solved for the maximum likelihood problem are given by

$$
\begin{gathered}
\overline{u^{2}} \Lambda=\frac{1}{N} \sum_{j=1}^{N} \frac{S_{j}}{F_{j}(\Lambda)} \\
\sum_{j=1}^{N}\left\{\left[\frac{d}{d \Lambda} \ln F_{j}(\Lambda)\right]\left[\frac{S_{j}}{F_{j}(\Lambda)}-\frac{1}{N} \sum_{i=1}^{N} \frac{S_{i}}{F_{i}(\Lambda)}\right]\right\}=0
\end{gathered}
$$

Indexes $i, j$ in $S_{i}, S_{j}, F_{i}$ and $F_{j}$ stand for the $i^{t h}$ or $j^{t h}$ frequency bin of the frequency vector where the estimated power spectral density $S(f)$ is evaluated. For simplicity, we keep the same notation was used by Mark [25]. $G_{i}$ and $F_{i}$ are auxiliary functions, which are described as follows

$$
G_{j}(\Lambda) \triangleq \frac{\mathrm{d}}{\mathrm{d} \Lambda} \ln F_{j}(\Lambda)
$$


and the solutions for each velocity component are given by

Longitudinal spectrum $S_{11}(f)$ :

$$
\begin{gathered}
F_{\hat{k}}(\Lambda) \equiv F(\hat{k}, \Lambda)=\frac{2}{U_{0}} \frac{2}{\left(1+\hat{k}^{2}\right)^{5 / 6}} \\
G_{\hat{k}}(\Lambda) \equiv \frac{\mathrm{d}}{\mathrm{d} \Lambda} \ln F(\hat{k}, \Lambda)=-\frac{2}{U_{0}} \frac{1}{\Lambda} \frac{\frac{5}{3} \hat{k}^{2}}{1+\hat{k}^{2}}
\end{gathered}
$$

Transverse spectrum $S_{22}(f)$ :

$$
\begin{gathered}
F_{\hat{k}}(\Lambda) \equiv F(\hat{k}, \Lambda)=\frac{2}{U_{0}} \frac{1+\frac{8}{3} \hat{k}^{2}}{\left(1+\hat{k}^{2}\right)^{11 / 6}} \\
G_{\hat{k}}(\Lambda) \equiv \frac{\mathrm{d}}{\mathrm{d} \Lambda} \ln F(\hat{k}, \Lambda)=\frac{2}{U_{0}} \frac{1}{\Lambda} \frac{\frac{5}{3} \hat{k}^{2}\left(1-\frac{8}{3} \hat{k}^{2}\right)}{\left(1+\hat{k}^{2}\right)\left(1+\frac{8}{3} \hat{k}^{2}\right)}
\end{gathered}
$$

The first step towards solving this problem ist to solve Eq. $21 \mathrm{~b}$, as it is a function only of the measured spectrum $S_{i} \triangleq S(f)$ and the von-Kármán model. Eq. $21 b$ is intrinsically a minimization problem that needs to be solved interactively. The outcome of it delivers the optimal turbulence integral length scale $\Lambda$, which is required as an input for the subsequent solution of Eq. 21a. An important aspect of this technique is that Eq. 21a tries to find the optimal $\overline{u^{2}}$ by matching the power of the modelled turbulence to the power of the estimated spectrum from experimental data $S(f)$. One significant disadvantage of this approach is that estimated parameters strongly deviate in the presence of tones or similar disturbances in the spectrum. As previously commented, the spectral fitting techniques described in Sections IV.C and IV.D are more robust and therefore more suitable for contaminated spectra. This difference in the technique will be further substantiated for the test cases using synthetic and experimental data.

\section{Synthetic test case}

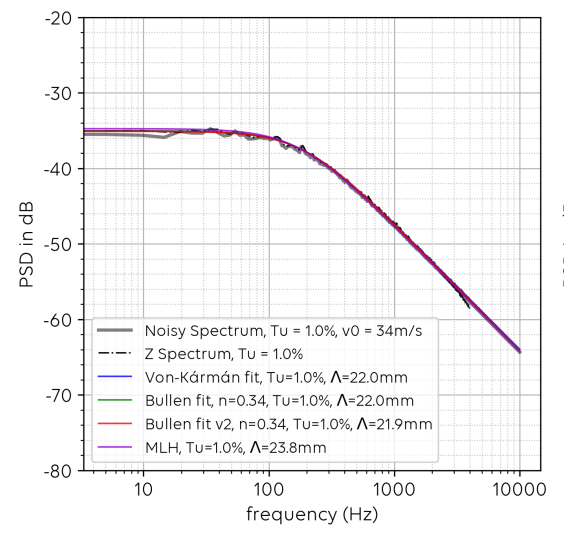

(a) Test case 1.

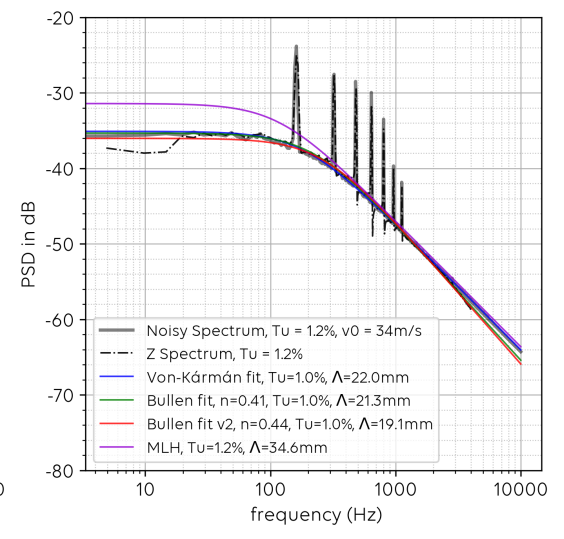

(b) Test case 2.

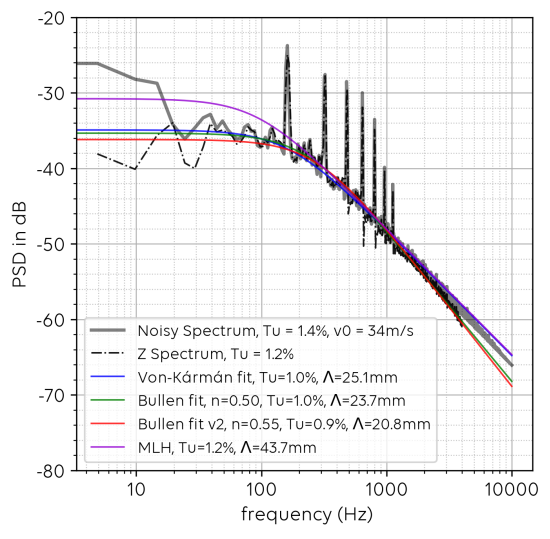

(c) Test case 3.

Fig. 3 Synthetic turbulence spectrum for the longitudinal velocity component. Parameters used: $U_{0}=\mathbf{3 4} \mathbf{~ m} / \mathbf{s}$, $T u=1 \%, I L S=20 \mathrm{~mm}$. Different sources of noise are added. Parameter estimation results are plotted with different colors.

In order to test the different parameter estimation techniques, the previously generated von-Kármán spectrum (Figure 1a, blue curve) is used as a reference spectrum, now superposed with noise to simulate the effects of spectral contamination on the prediction capabilities of the proposed methods. Three different sources of noise are added:

1) White noise with $\sigma=2$ and low-passed with cut-off frequency set by $250 \mathrm{~Hz}$ was directly added to the spectrum (see Fig. 3a.

2) Harmonic tones up to the 7th order with decreasing amplitude combined with (1) were added aiming to simulate BPF tones in the spectrum, often seen in turbulence measurements in turbo-machinery [31, 32], shown in Fig. $3 \mathrm{~b}$ 
3) Low-frequency disturbance: Around $9 \mathrm{~dB}$ noise amplitude was added directly to spectrum in the frequency range from 0 to $17 \mathrm{~Hz}$. This source of disturbance is not easy to reproduce, as it has a non-Gaussian origin [28, 33]. In this test however, a simply addition in the low-frequency range was performed. The combination of all these 3 sources of disturbances are shown in Fig. 3c

After the addition of these distortions, each spectrum was again normalized with the power of the original one (noise free), in order to roughly conserve its power. This source spectrum is shown as the gray thick curves in Fig. 3 labeled as "Noisy Spectrum". The dashed black curve ("Z Spectrum") represents the output spectrum after the low-frequency removal technique. This spectrum is used as input for all parameter estimating techniques. In Fig. 3 blue curve (von-Kármán model) and green curve (Bullen model) were fitted according to the formulation described in Section 19 The red curve (Bullen model) was fitted with constraint, as shown in Section IV.D Purple curve stands for the result obtained with the Maximum Likelihood technique, described in Section IV.E

It is worth mentioning, that after the addition of all distortions, the original parameters such as turbulence levels and integral length scale are not expected to match the initial ones, as the additional noise changes the energy content and shape of the spectrum. The Maximum Likelihood technique converged to different values when compared to all three fit techniques. In Figs. $3 \mathrm{~b}$ and $3 \mathrm{c}$ the purple curve is clearly higher than the other curves. This error is due to the presence of tones in the spectrum.

This test helps to show how each technique performs under different distortions present in the signal. Further, the fit technique seems to be robust against the existence of tones in the spectrum, as discussed in Section IV.C

\section{Hot-wire measurement setup}

The aerodynamic database presented in this work was obtained at AneCom AeroTest, at the Universal Fan Facility for Acoustics (UFFA) of AneCom AeroTest in Wildau, Germany. The test campaign was conducted in the frame of the EU project TurboNoiseBB. Tests were carried out on the ACAT1 transonic fan, with a design pressure ratio of about 1.42 and a corresponding bypass-ratio of around 8 . The rotor has 20 blades. The modular fan rig diameter is 34 inches $(864 \mathrm{~mm})$ and is powered by an $18 \mathrm{MW}$ motor.

The hot-wire probe with two wires was mounted in a radial traverse (Fig. 4a) enabling measurements ranging from $5 \mathrm{~mm}$ to $160 \mathrm{~mm}$ inside the channel. Fig. $4 \mathrm{~b}$ shows a cross-section of the test rig. The red line shows roughly the radial path of the hot-wire probe and the axial position of the measurement plane. More information about the DLR's hot-wire measurement capabilities can be found in [16, 31, 32].

For each measurement point, 10 seconds of data, sampled at $192 \mathrm{kHz}$, were acquired. A 1 pulse-per-rev trigger signal was simultaneously acquired. In the frame of the current work, two operating points were selected and analyzed: $\eta_{1} \approx 3800 \mathrm{RPM}$, with $U_{0} \approx 83 \mathrm{~m} / \mathrm{s}(\mathrm{M}=0.24)$ and $\eta_{2} \approx 6840 \mathrm{RPM}$, with $U_{0} \approx 166 \mathrm{~m} / \mathrm{s}(\mathrm{M}=0.48)$.

\section{Experimental results}

This section starts with the assessment of each parameter estimation technique with respect to the experimental data. Measurements inside the boundary-layer (higher turbulence levels, lower noise ${ }^{\text {W) }}$ and outside (lower turbulence levels, higher noise) are used. The spectra estimated from measured data were limited to a frequency up to $4 \mathrm{kHz}$ for all cases, which were then used as input for all parameter estimation techniques.

\section{A. Power spectral density}

Fig. 5 shows a test case for measurements performed inside the turbulent boundary layer. The same color map as in previous figures is used. The thick gray curve shows the estimated spectrum from raw velocity data. The estimated turbulence intensity and turbulence integral length scale are plotted in the legend of each figure. The black curve represents, as previously, the spectrum after a cyclostationary analysis, low-frequency components removal and band-limited up to $4 \mathrm{kHz}$.

The raw spectrum is clean for $\eta_{1}$, but for $\eta_{2}$ spurious noise starts emerging for $\mathrm{f}>2 \mathrm{kHz}$. Despite this, all techniques converged to similar values of turbulence levels and integral length scale. The Maximum Likelihood (MLH) technique deviates the most compared to the fit techniques. It is also worth to further discuss the decay of the spectrum at high frequencies. The gradient of the experimental spectrum is significantly steeper than the blue and purple curves (von-Kármán spectrum). Different reasons can be associated to this phenomenon: If the decay is indeed related to the

\footnotetext{
${ }^{*}$ in this context noise is used to denote all components that are not turbulence related, such as tones, low-frequency disturbance, etc.
} 


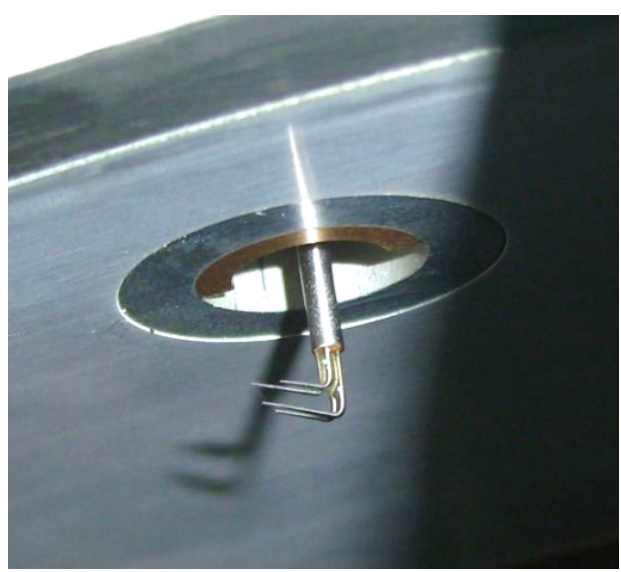

(a) Photograph of the two velocity components u,v hot-wire probe.

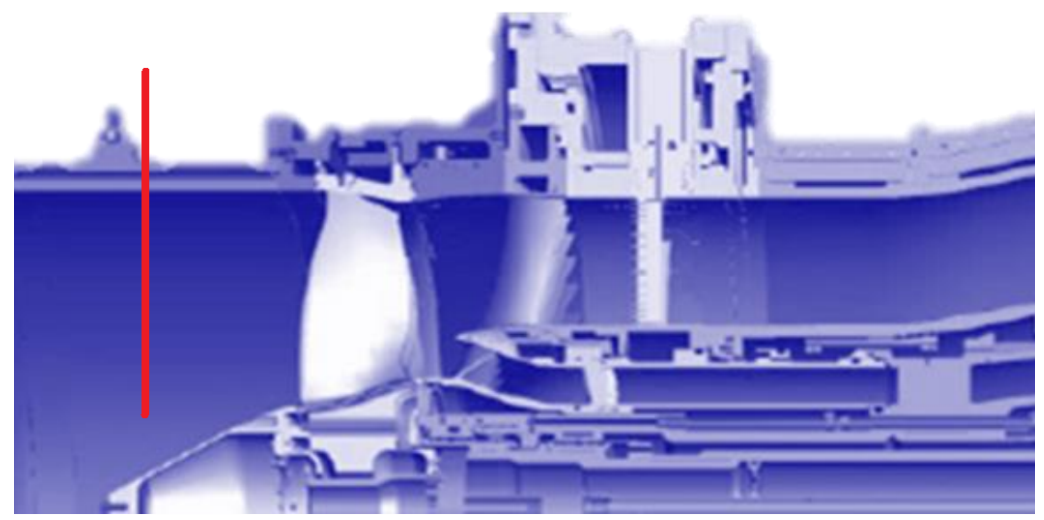

(b) UFFA test rig cross-section [34]. Red line roughly indicates the axial position of the hot-wire probe in the fan inflow section.

\section{Fig. 4 Detailed of the hot-wire instrumentation and the UFFA test rig cross-section.}

turbulence, then the von-Kármán spectrum fails to fully capture the measured data. On the other hand, this steeper gradient can be related to the measurement technique, more specifically the diameter of the hot-wires. Polacsek et al. [14] showed for the same data-set, that this steeper gradient could be explained by the limited band-width of the hot-wires, resulting in a poor resolution of high-frequency velocity fluctuations. In other words, the steeper decay could be attributed to artifacts from the measurements rather than be related to the physics of turbulence. Since no systematic study regarding the effect of the wire thickness was conducted, some uncertainty regarding the observed differences in slope at higher frequencies remains.

Fig. 6 depicts results for measurements outside the boundary layer, where especially strong low-frequency components are present in the signal. In spite of that, all techniques converged to comparable results for the longitudinal component. Note that the turbulence intensity of the raw spectrum is roughly $2 \%$, whereas all other methods predict a turbulence intensity of about $1 \%$. This means, if no signal conditioning is applied to the data, an error of $100 \%$ in the estimation of turbulence intensity would be made. For the transverse component, the MLH and "fit v2" techniques did not yield satisfactory results. As a plateau can be theoretically expected at lower frequencies in the spectrum, the post-processed "Z spectrum" likely still contain some contamination, which at least partly explains the poor performance of the previously mentioned techniques. The estimated turbulence intensity is again about half of the value obtained from the raw data. 


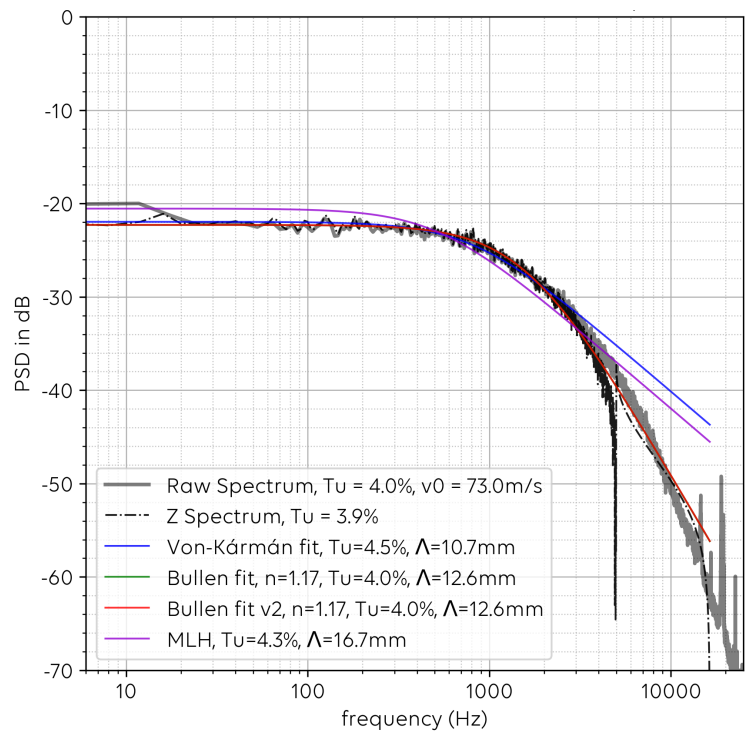

(a) $\eta_{1} \approx 3800$ RPM

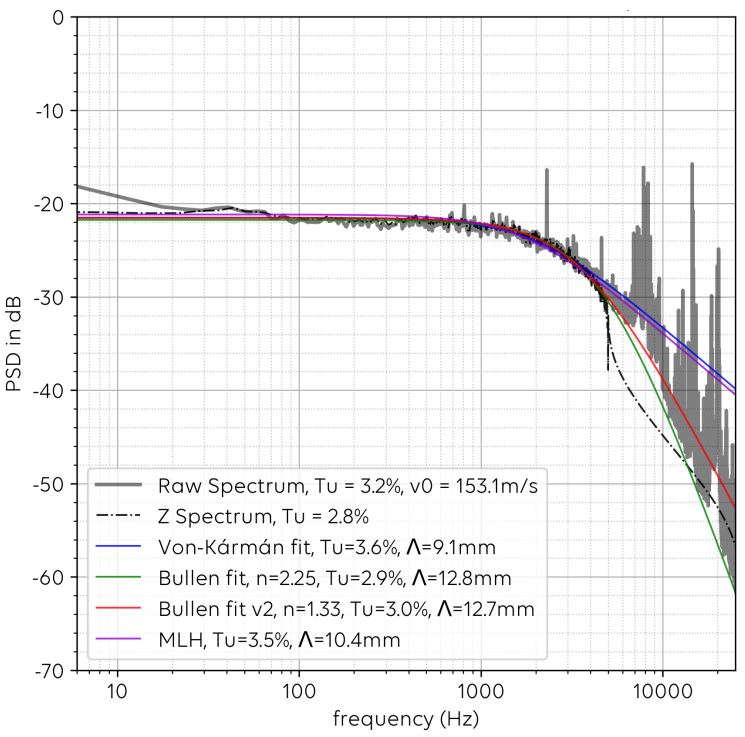

(b) $\eta_{2} \approx 6840 \mathrm{RPM}$

Fig. 5 Test case for measurements performed inside the turbulent boundary layer. Both plots regard to the longitudinal velocity component.

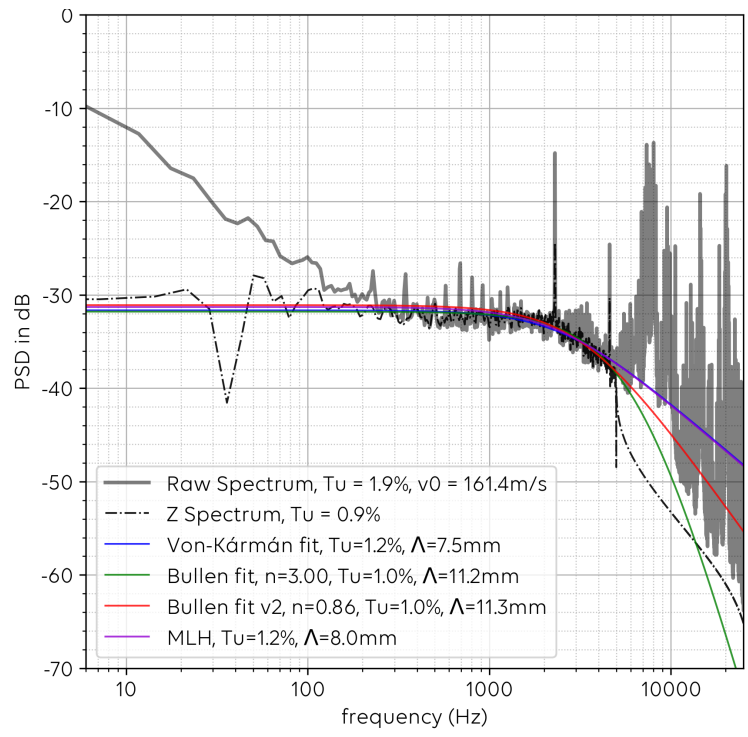

(a) Longitudinal velocity component.

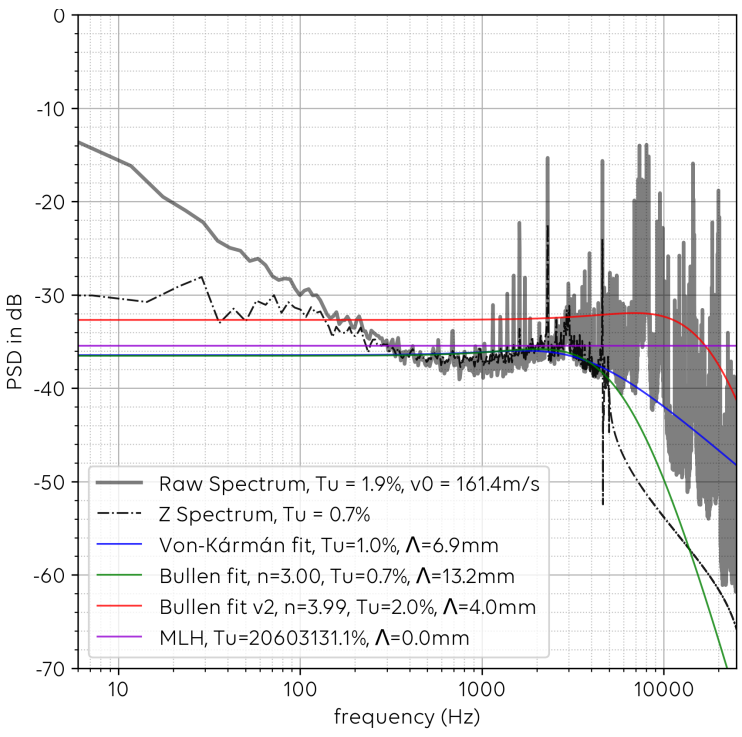

(b) Transverse velocity component.

Fig. 6 Measurement outside the turbulent boundary layer for $\eta_{2} \approx 6840$ RPM. Strong disturbances are seen at low frequencies: $\mathrm{f}<100 \mathrm{~Hz}$ and for $\mathrm{f}>5 \mathrm{kHz}$.

\section{B. Results for several probe inserts}

A collection of selected results for 5 different probe insertions are plotted in Fig. 7 The normalization of the spectra enables a direct comparison of the spectral shapes from different measurement points. It is also a rough way of validating whether the parameters integral length scale and turbulence intensity are (approx.) correct. A von-Kármán model is also plotted as a reference, since the von-Kármán spectrum is commonly used to describe turbulence in turbo-machinery. For the lower speed case of $\eta_{1} \approx 3800$ RPM (see Figs. $7 \mathrm{a}$ and $7 \mathrm{~b}$ ), the spectra obtained at measurement positions within the turbulent boundary layer $(5,10$ and $30 \mathrm{~mm})$, all spectra are quite similar except for the previously discussed 

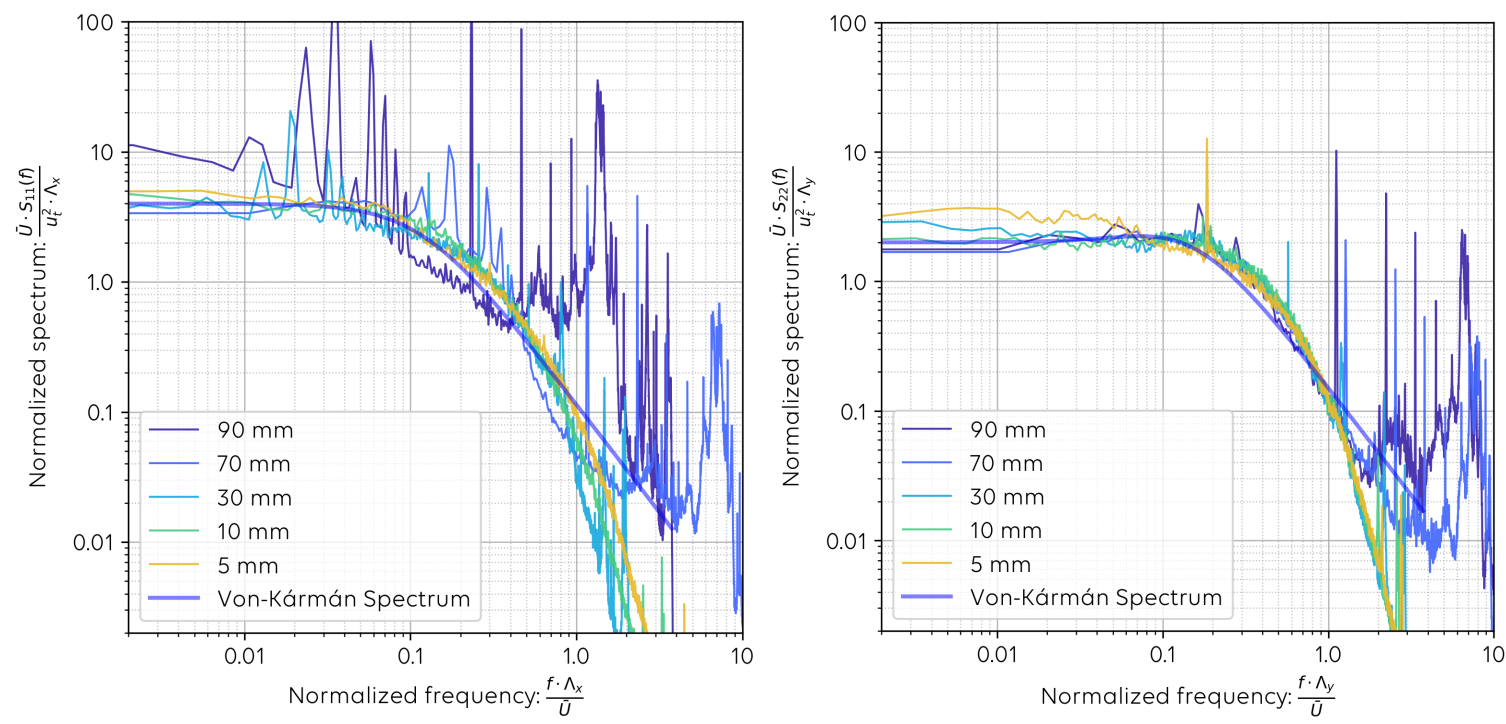

(a) Normalized turbulence spectrum $S_{11}(f)$ for different probe insert positions. $\eta_{1} \approx \mathbf{3 8 0 0} \mathbf{R P M}$.

(b) Normalized turbulence spectrum $S_{22}(f)$ for different probe insert positions. $\eta_{1} \approx 3800$ RPM.
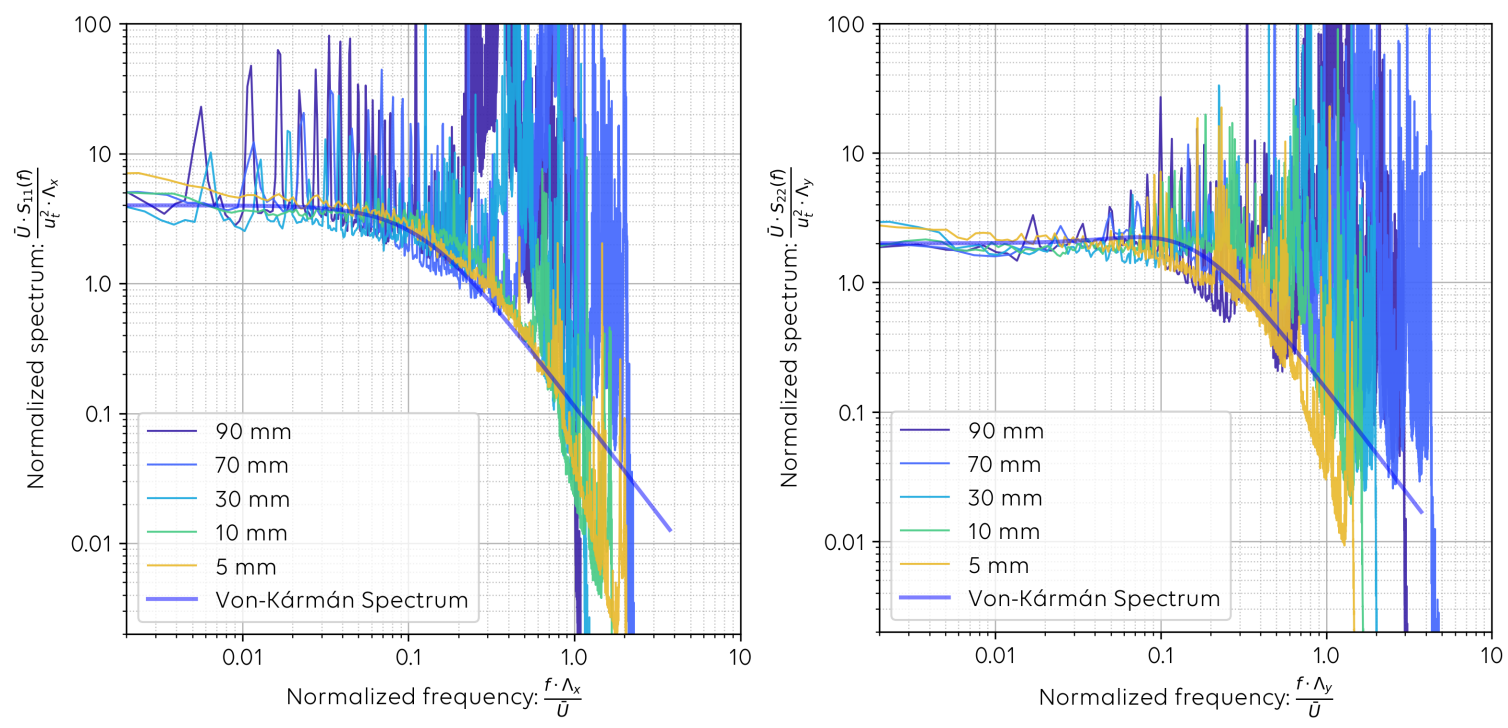

(c) Normalized turbulence spectrum $S_{11}(f)$ for different probe insert positions. $\eta_{2} \approx 6840 \mathrm{RPM}$.

(d) Normalized turbulence spectrum $S_{22}(f)$ for different probe insert positions. $\eta_{2} \approx 6840 \mathrm{RPM}$.

Fig. 7 Results obtained for selected measurement points and two fan rotation speeds. Numbers in the legend refer to the distance from wall (probe insertion). Spectra are normalized by the estimated parameters $\overline{u^{2}}, \Lambda_{1}$. A von-Kármán spectrum is also plot as reference guide, at which all spectra should roughly fall.

discrepancies at high frequencies. The tones and distortions present in the signal, especially for the measurements outside the turbulent boundary layer $(70$ and $90 \mathrm{~mm}$ ), not only make it harder to estimate turbulence parameters, but it is also more difficult to judge the similarity in spectral shapes, whether every normalized spectrum coincides with the other spectra. When all of the spectra roughly fall together, this means that the flow parameters should (potentially) be estimated correctly.

Figs. $7 \mathrm{c}$ and $7 \mathrm{~d}$ depict the same probe insertions and velocity components, but now for a higher fan speed $\eta_{2} \approx 6840$ RPM. Distortions in the signal are stronger for this fan speed. Nonetheless, all spectra roughly collapse together. The spectra decay is again steeper than the von-Kármán spectrum and the same possible reasons as previously mentioned 
hold for this case as well.

On Fig. 8 the turbulence intensity profile is plotted alongside the normalized axial velocity, whereas the latter serves as a reference. Both Figs. $8 \mathrm{a}$ and $8 \mathrm{~b}$ show the longitudinal velocity component. On the legend the label "improved" means the turbulence estimated from the "Z spectrum", without any further turbulence spectrum modeling. All techniques converged to a narrow range of values. For both fan speeds, the raw data resulted in much higher turbulence intensity value, which can be explained by the high level of contamination in the raw signal. This is particularly evident for $\eta_{2}$ on Fig. $8 \mathrm{~b}$, where the difference compared to all estimators is enormous.

Fig. 9 shows the turbulence integral length scale, which was computed from the longitudinal and transverse fluctuating velocity components for both fan speeds. The thick curve (blue and red) shows the more likely value, which was determined by analyzing the spectral fits of all techniques. The shaded range serves as an estimate of an interval of confidence, since it contains all predicted integral length scale values outputted by the various techniques. Besides previously applied techniques, the integral length scale calculated from the "Z spectrum" via Eq. 11 and from raw data via Eq. 9 are also included. For measurements close to the duct wall, where the turbulence intensity is much higher and very low noise are present, all techniques converge to similar values. However, the confidence interval increasingly widens due to an increase in signal contamination as the probe is further immersed in the flow.

Since the fitting techniques were applied with respect to the longitudinal and transverse fluctuating velocity components, differences in the estimates can either indicate that the assumption of HIT is less applicable in certain regions of the flow or that the longitudinal and transverse spectra contain significantly different levels of contamination. Fig. 10] shows the turbulence intensity ratio of the both velocity components and the integral length scale ratio. In the case of HIT, the thick, red lines indicate the expected ratios. For both turbulence intensity and integral length scale, the measured turbulence does not seem to be isotropic at any measurement position. It is worth mentioning, that for higher probe insertions, the integral length scale of the transverse component has values ranging from approx. 2 to 5 times the longitudinal component, which is far away from isotropic turbulence.

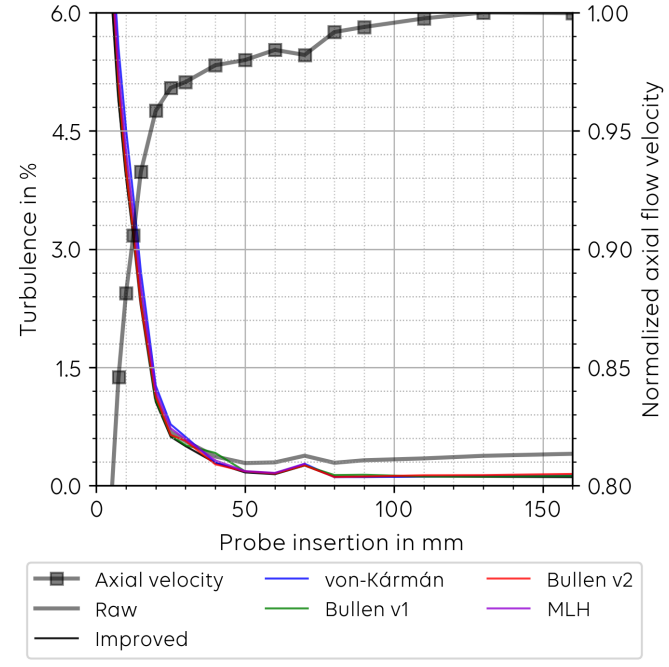

(a) Turbulence intensity with respect to the longitudinal velocity component. $\eta_{1} \approx 3800$ RPM.

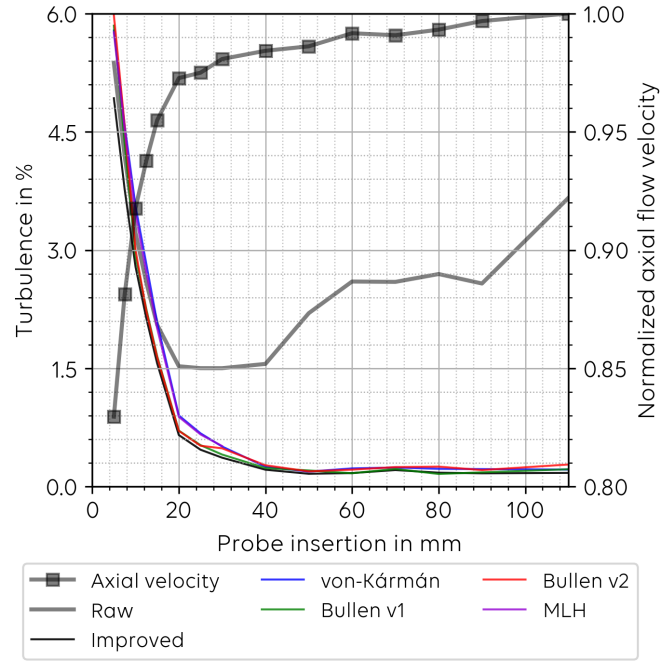

(b) Turbulence intensity with respect to the longitudinal velocity component. $\eta_{2} \approx 6840 \mathrm{RPM}$.

Fig. 8 Turbulence intensity result obtained for all estimator techniques, several probe insert depth and for two different fan speeds.

\section{Conclusions}

This work has compared several different techniques to extract turbulence information such as integral length scale ILS and turbulence intensity (or turbulent kinetic energy - TKE) from noise free and strongly contaminated hot-wire velocity measurements. The turbulence spectra von-Kármán and Bullen (Fig. 11) were used to determine turbulence parameters from contaminated measurement data. The difference between the Bullen model over the von-Kármán is that the Bullen model features an adaptable gradient at high frequencies. 


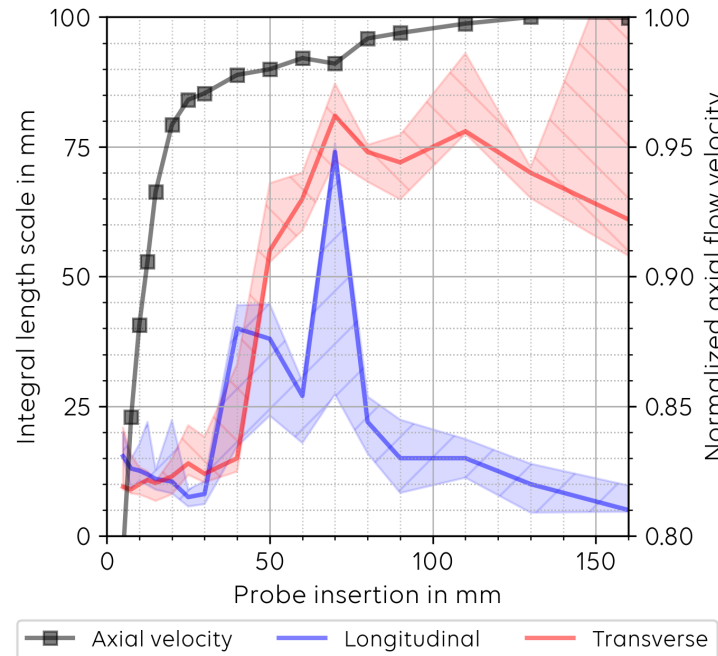

(a) Results for $\eta_{1} \approx 3800$ RPM.

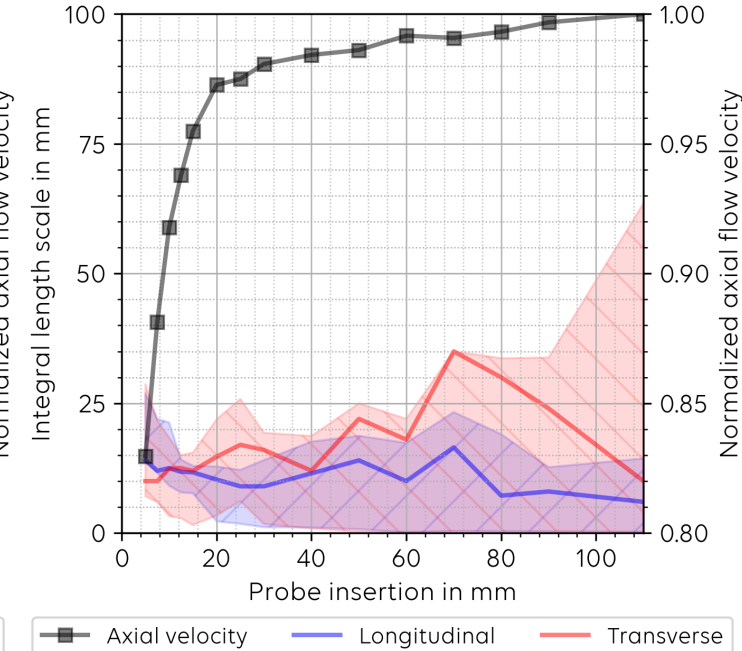

(b) Results for $\eta_{2} \approx 6840$ RPM.

Fig. 9 Turbulence integral length scale predicted with respect to the longitudinal and transverse velocity component and for two fan speeds. The shaded regions roughly indicate the confidence interval.

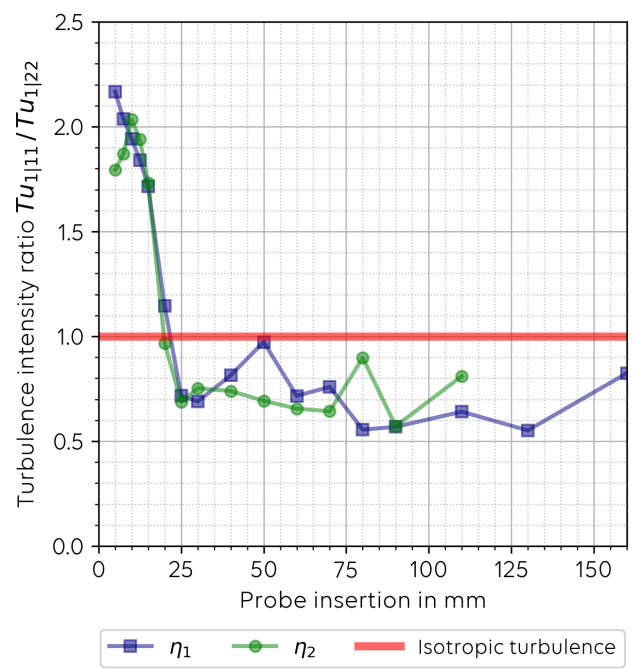

(a) Turbulence intensity ratio of the longitudinal and transverse velocity component.

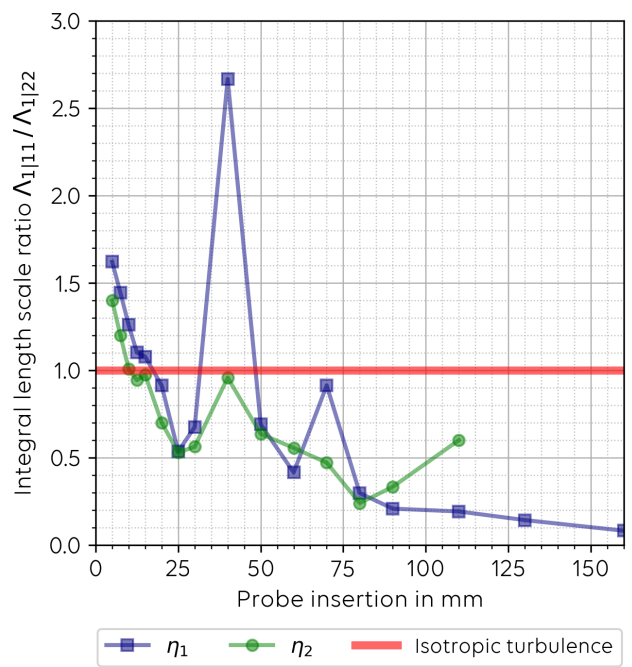

(b) Turbulence integral length scale ratio of the longitudinal and transverse velocity component.

Fig. 10 Turbulence isotropy assessment for two different fan speeds: $\eta_{1} \approx 3800 \mathrm{RPM}$ and $\eta_{2} \approx 6840 \mathrm{RPM}$ and different hot-wire probe insertion positions. The thick red line shows where the respective value for isotropic turbulence should lie.

For the first synthetic test case (Fig. 3) without distortions, all techniques performed similarly and determined the turbulence parameters correctly. For the second test case however, when harmonic tones were added to the spectrum, the performance of the maximum likelihood technique was negatively influenced. This can be explained by the fact that this technique tries to match the power of the modelled spectrum to the input spectrum (in this case, the test case spectrum). Therefore, if components that are known to not be turbulence related, such as tones, will bias the estimated turbulence intensity. Meanwhile all the proposed spectral fit techniques performed well for both estimating the original turbulence intensity as well as the integral length scale. In the third test case, Fig. 3c. with stronger white noise and low-frequency noise added, the low-frequency removal technique proved to work, as well the spectral fitting techniques. The maximum 
likelihood was again biased by the strong tones present in the spectrum leading to errors in the parameter estimation.

When tested with measured data inside the turbulent boundary layer (Fig. 55, where turbulence intensity is high and distortion levels are low, all techniques performed similarly. The Bullen spectrum captured more accurately the experimental spectral decay, whereas the von-Kármán spectrum features a fixed decay rate, which is not as steep as for the experimental spectra. Whether this is indicator for the better suitability of the Bullen turbulence spectrum is not clear, as the decay may not be related to the turbulence physics as discussed in Section VII.A The following test with the hot-wire element inside the free-stream (outside of the turbulent boundary layer, as in Fig. 6) exhibited strong contamination as discussed for the synthetic test case: strong low-frequency components, tonal components possibly due to probe and/or wire vibration, etc. The cyclostationary analysis could remove most of the tones present in the raw data and the low-frequency removal technique on removing the low-frequency part of the spectrum, with respect to the "slow" part of the auto-correlation function.

When several probe insertions are analyzed and plotted together as normalized spectra as in Fig. 7, all of them feature a similar von-Kármán-like spectral shape despite the strong contamination contained in the spectra. This analysis shows that while the estimated parameters are not necessarily precisely correct, but likely within the same order of magnitude of the "true values".

As a matter of comparing side-by-side all technique for estimating the turbulence intensity, Fig. 8 portrayed the result for the longitudinal component for two different rotor speeds. All techniques fell approx. in the same range. This could be contrasted with the value obtained from the raw spectrum. Especially for the higher speed, the raw turbulence intensity was over $3 \%$ inside the channel, whereas the models showed that it was expected to be bellow $0.3 \%$, one order of magnitude difference.

Finally, an isotropy assessment was attempted by analyzing the turbulence intensity and integral length scale ratio predicted using longitudinal and transverse velocity components along several hot-wire insertion positions, as shown in Fig. 10 As observed, the flow seem not to be isotropic, especially when outside the turbulent boundary layer, where the turbulence length scale in the transverse direction reached up to 4 times the one in the longitudinal direction. The turbulence ratio of both components also did not reach value 1 (1 would indicate same level of turbulence intensity on both velocity components). Indeed inside the turbulent boundary layer the turbulence intensity in the longitudinal direction is stronger than in the transverse, while outside this boundary, the opposite happens.

Despite using isotropic and homogeneous turbulence spectrum models (von-Kármán and Bullen) to assess potentially non-isotropic flow, results obtained in this work seem plausible and allowed the estimation of flow parameters with a reasonable precision.

\section{Acknowledgments}

The presented AneCom AeroTest 1 data were obtained in the frame of the project TurboNoiseBB, which has received funding from the European Union's Horizon 2020 research and innovation program under grant agreement number 690714.

\section{References}

[1] Fransson, J. H. M., "Free-stream turbulence and its influence on boundary-layer transition," Tenth International Symposium on Turbulence and Shear Flow Phenomena. Begel House Inc., 2017.

[2] Butler, e. a., Robert J., "The effect of turbulence intensity and length scale on low-pressure turbine blade aerodynamics," International journal of heat and fluid flow 22.2 (2001): 123-133., 2001.

[3] Moss, R. W., “The effects of turbulence length scale on heat transfer.” Ph.D. thesis, University of Oxford, 1992.

[4] Moss, R. W., and Oldfield., M. L. G., "Measurements of the effect of free-stream turbulence length scale on heat transfer," ASME 1992 International Gas Turbine and Aeroengine Congress and Exposition., 1992.

[5] Leonard, T., Sanjose, M., Moreau, S., and Duchaine, F., "Large Eddy Simulation of a scale-model turbofan for fan noise source diagnostic,” 22nd AIAA/CEAS Aeroacoustics Conference, AIAA 2016-3000, 2016.

[6] Zenger, F., Herold, G., , and Becker, S., "Acoustic characterization of forward-and backward-skewed axial fans under increased inflow turbulence," AIAA Journal 55.4 (2017): 1241-1250., 2017.

[7] Zenger, F. J., Renz, A., Becher, M., and Becker, S., "Experimental investigation of the noise emission of axial fans under distorted inflow conditions," Journal of Sound and Vibration 383 (2016): 124-145, 2016. 
[8] Staggat, M., Moreau, A., and Guérin, S., "Analytical prediction of boundary layer ingestion noise for an integrated turbofan," Proceedings of the 26th International Congress on Sound and Vibration, ICSV 2019., 2019.

[9] Moreau, A., "A unified analytical approach for the acousticconceptual design of fans of modern aero-engines," Ph.D. thesis, Technische Universität Berlin, 2017.

[10] Guérin, S., and et al., "Noise prediction of the ACAT1 fan with a RANS-informed analytical method: success and challenge," AIAA 2019-2500, 25th AIAA/CEAS Aeroacoustics Conference, Delft, Netherlands, May 20-24, 2019, 2019.

[11] Lewis, D., Moreau, S., and Jacob., M., "On the use of Large Eddy Simulations and analytical models to perform broadband rotor-stator interaction noise predictions." 25th AIAA/CEAS Aeroacoustics Conference, Delft, Netherlands, May 20-24, 2019, 2019.

[12] Kissner, C., and Guérin, S., "Fan Broadband Noise Prediction for the ACAT1 Fan Using a Three[1]Dimensional Random Particle Mesh Method," 26th AIAA/CEAS Aeroacoustics Conference, 2020. URL https://doi.org/10.2514/6.2020-2520, 2020.

[13] Kissner, C., and Guérin, S., "Comparison of Predicted Fan Broadband Noise Using a Two-versus a Three-Dimensional Synthetic Turbulence Method," Journal of Sound and Vibration (2021): 116221, 2021.

[14] Polacsek, C., Cader, A., Barrier, R., de Laborderie, H., and Gea-Aguilera, F., "Aeroacoustic design and broadband noise predictions of a turbofan stage with serrated outlet guide vanes." 26th International congress on sound and vibration, Montreal, 7-11 July, 2019.

[15] Lewis, D., Moreau, S., and Jacob, M., "Broadband Noise Predictions on the ACAT1 Fan Stage Using Large Eddy Simulations and Analytical Models," 26th AIAA/CEAS Aeroacoustics Conference, virtual, 2020. doi: 10.2514/6. 2020-2519., 2020.

[16] Meyer, R., Hakansson, S., Hage, W., and Enghardt, L., "Instantaneous flow field measurements in the interstage section between a fan and the outlet guiding vanes at different axial positions." Proceedings of the 13th European Conference on Turbomachinery Fluid Dynamics and Thermodynamics, Lausanne, Switzerland., 2019.

[17] Tennekes, H., and Lumley, J. L., A First Course in Turbulence, The MIT Press, 1972.

[18] Hinze, J. O., Turbulence, McGraw-Hill series in mechanical engineering, 1975.

[19] Richardson, L. F., Weather prediction by numerical process, Cambridge university press, 2007.

[20] Percival, D. B., and Walden, A. T., Spectral analysis for physical applications, Cambridge University Press, 1993.

[21] Batchelor, G. K., The Theory of Homogeneous Turbulence, Cambridge University Press, New York, 1953.

[22] Roach, P. E., "The generation of nearly isotropicturbulence by means of grids," International Journal of Heat and Fluid Flow 8.2: 82-92., 1987.

[23] Wohlbrandt, A., "Stochastisches Verfahren zur Simulation von Breitbandschall in Triebwerkfans," Ph.D. thesis, Technischen Universität Berlin, 2017.

[24] Mark, W. D., "Methods for estimation of Bullen turbulence spectrum parameters," NASA-CR-165912, 1982.

[25] Mark, W. D., “Maximum Likelihood Estimationof Turbulence Spectrum Parameters,” AIAA Journal, VOL. 22, NO. 1, 1982.

[26] Behn, M., Pardowitz, B., and Tapken, U., "Separation of Tonal and Broadband Noise Components by Cyclostationary Analysis of the Modal Sound Field in a Low-speed Fan Test Rig," International Conference of Fan Noise, Aerodynamics, Applications and Systems, 18-20 April 2018, Darmstadt, Germany, Paper No. 43., 2018.

[27] Antoni, J., “Cyclostationarity by examples,” Mechanical Systems and Signal Processing 23.4 (2009): 987-1036., 2009.

[28] Mark, W. D., "Characterization, Parameter Estimation, and Aircraft Response Statistics of Atmospheric Turbulence," NASA Contractor Report 3463, 1981.

[29] Kay, S. M., Fundamentals of statistical signal processing, 1993.

[30] Pope, S. B., Turbulent Flows, Cambridge University Press, 2000.

[31] Meyer, R., Knobloch, K., and Hakansson, S., "Hot-wire measurement in a Direct Driven high speed Turbo Fan (DDTF) Rig," ETC-11-256. 11th European Turbomachinery Conference; March 23-27, 2015, Madrid, Spain., 2015. 
[32] Meillard, L., Schnell, R., Meyer, R., and Voigt, C., "Time resolved pressure and velocity measurements at the DLR UHBR-Fan and comparison with simulation data," 62. Deutscher Luft- und Raumfahrtkongress, 10.-12. September 2013, Stuttgart, Germany., 2013.

[33] Mark, W. D., and Fischer, R. W., "Investigation of the effects of nonhomogeneous (or nonstationary) behavior on the spectra of atmospheric turbulence.” Tech. rep., NASA CR-2745, 1976.

[34] Tapken, U., Bauers, R., Neuhaus, L., Humphreys, N., Wilson, A., Stöhr, C., and Beutke, M., “A New Modular Fan RigNoise Test and Radial Mode Detection Capability," 17th AIAA/CEAS Aeroacoustics Conference, Portland, Oregon (USA), June 2011, pp. AIAA 2011-2897, 2011. 